\title{
The Decision Arena: A model-centric interactive workspace for Product-Service System design
}

\author{
Johan Wall ${ }^{1}$, Marco Bertoni ${ }^{1}$ \\ ${ }^{I}$ Blekinge Institute of Technology \\ johan.wall@bth.se \\ marco.bertoni@bth.se
}

\begin{abstract}
A new product development landscape arises from the increasing competition of the global markets, emphasizing the need for a more collaborative process, both within and outside the organization. Design has become an intense 'social activity': working with others and sharing a common understanding are of critical importance to find satisfying solutions for customers and stakeholders. This leads to the issue of how to set up a collaborative, model-based physical environment to foster collaboration and knowledge sharing when different disciplines get together in the 'design exercise'. This paper describes the development of such an environment, named the 'Decision Arena' or DA. This mimics a war-room for Product-Service System design, where the physical space and dedicated hardware/software meet to support collocated collaborative decision making. The objective of the paper is to describe the physical and technical features of the existing DA prototype. It further presents the results of an experimental study involving a cross-disciplinary team of participants working in the road construction sector. Both the verbal communication and physical interaction measured in the experiment show that all participants took an active part in addressing the design challenge. This is seen by the researchers as an indication that the proposed model-centric environment for interactive group work has the potential to augment collaborative design and the mutual sharing of knowledge and values.
\end{abstract}

\section{Keywords: Decision Arena, Product-Service Systems, product development, model-centric}

\section{Introduction}

The knowledge needed to make well-informed decisions in engineering design rarely resides in a single person or organizational role. Product development activities have long been characterized by parallel workflows and by a concurrent engineering approach. This means for the design team to embrace an enterprise-wide view in decision making, including expertise from procurement, supply chain, manufacturing, marketing, and more (Wang et al. 2002). The increasing complexity of products and the transition towards Product-Service Systems (PSS) fuel, even more, the need to share knowledge and values across disciplines and company functions (Sethi, 2000; Murakami, 2016). The complexity of engineered systems has increased rapidly over the past decades driven by the rapid development of the Internet of Things (IoT) and information technology in general. Modern mechatronic systems heavily rely on embedded 
software to fulfill functionality, asking for tighter integration between the mechanical and software engineering team. Furthermore, PSS-like business approaches emphasize the need to co-develop product and service bundles based on product life extension (often through sharing), de facto dematerializing the physical artifacts (Bey \& Mcaloone, 2006). Hence, product development can be described today as a complex ambiguity game. A wide variety of expert competencies, transcending traditional disciplines, are needed to evaluate the impact of decisions in the early phases of PSS design. By early phases the authors mainly refer to concept development and system-level design according to the model presented by Ulrich and Eppinger (2012). A common approach to deal with complexity is to transform 'complex' into 'manageable and controllable' through the use of modeling and simulation (ElMaraghy et al., 2012). By means of simplified descriptions of reality, the cross-functional team can evaluate and explore (through simulation) complex problems in a resource-efficient way (Brown, 2015). The phenomena described above show a clear incentive towards involving a broad range of stakeholders into the decision process, both internally in an organization and externally (mainly suppliers and customers). Gathering a diverse group of individuals in a collaborative setting is observed to increase the effectiveness of the early-stage design exploration exercise, which is typically characterized by scarce and even conflicting information. A model-centric environment for collaborative decision making, the Decision Arena (DA), has previously been proposed as a solution that may help to harvest these benefits (Wall et al., 2018). The objective of this paper is to present the evolution of the DA concept, to describe its infrastructure, and to presents the results of experimental activities conducted with industrial practitioners. The underlying research question of this work can be described as the following:

RQ: How should a model-driven interactive group workspace be designed and equipped to invite and support collaboration within a cross-functional design team.

The paper initially describes the rationale of the DA environment, detailing its main functions, architectural components, and models. The paper further illustrates the results of verification activities conducted with industrial practitioners on the initial DA prototype.

\section{Research method}

The Design Research Methodology (DRM) proposed by Blessing and Chakrabarti (2011) was used as the main reference throughout the research. After conducting the initial Research Clarification (RC) stage, the authors undertook a comprehensive Descriptive Study I (DS-I) to obtain a sufficient understanding of the user needs and preferences for the environment, which eventually drove the definition of the functionalities and requirements for a prototype in the Prescriptive Study (PS) phase. In the DS-I, relevant contributions were collected through a systematic search in the Scopus and Google Scholar databases and further complemented through snowballing (Wohlin, 2014) to find additional literature.

Noticeably, the empirical data gathering activities in DS-I are based on a multiple case study approach (Yin, 2011). This was considered to be relevant given the objective of developing a better understanding of the functions associated with the decision environment, and of demonstrating it across a range of design episodes. Several case studies were selected in advance and conducted at the same time, requiring the participation of several researchers or investigators. The cases range from aerospace applications to construction equipment, from mobility devices to food packaging. The initial Descriptive Study II (DS-II) was conducted through qualitative and quantitative means. Initially, the prototype environment was demonstrated to company practitioners and other industry experts, collecting their verbal feedback in the form of meeting minutes. Later, an experiment was set up to gather more factual 
data on the ability of the environment to support decision making. The experimental setup and its results are discussed in detail in Section 4.

\section{The Decision Arena}

Recent years have seen a surge of interest in the development of interactive group workspaces focused on digital workflows (Wigdor et al. 2009, Haller et al. 2010, Nieminen et al. 2013, Benyon \& Mival 2015). Yet, research contributions in the field of model-centric interactive workspaces are still limited, with the Decision Theatre (DT) at Arizona State University being one of the few exceptions (White et al. 2015). While the DT is a remarkable example of how to raise awareness of the macro-scale effect of design decision making, many (e.g., Rhodes \& Ross 2016) have expressed the need for more specific model-centric environments for engineering design. Wall et al. (2018) recently described the findings from initial work on conceptualizing, developing, and testing physical spaces for collocated collaborative decision making. This environment, named 'Decision Arena' (DA) is inspired by the concept of 'warroom' for PSS design. The underlying idea of the DA is to gather all relevant people in a single location, so as to improve the decision-making process, making sure that what is being decided is the 'right thing' to do. In this respect, the concept of 'value' is used as a proxy to measure the 'goodness' of a decision. This means that design trade-offs in the environment are assessed from the perspective of how much customers 'value' certain capabilities of a product/system against each other (Isaksson et al. 2015). Design proposals are evaluated by playing out potential usage scenarios and investigating cause and effect relationships, so as to build a shared understanding of the task at hand. The design team can play out different scenarios by manipulating models through graphical user interfaces, enabling also non-expert to actively participate. This work mode is centered around the use of digital models, simulation data and product data collected in the field. The DA may be described as a blended space (Benyon \& Mival, 2015) whose main value resides in the interaction between the physical and the digital world, meaning that the combination of the two creates a new space with its own emergent properties. Transiting from deterministic to probabilistic simulations to evaluate different operational scenarios is further considered to help decision-makers in identifying more robust and value-adding solutions. The interaction triggered by the DA is seen to be beneficial also to trigger trade-off negotiations continuing beyond the co-located exploration sessions.

\subsection{The DA physical space}

Figure 1 illustrates the current prototype of the Decision Arena. The opportunity of inviting collocated collaboration is the main principle guiding the design of the physical space. According to Craft \& Garcia (2016), face-to-face communication is the best vehicle for the conveyance of complex information including facial expression, gestures, and tone of voice. For this reason, the physical space is designed for collocated collaborative activities in small groups, typically consisting of five or fewer individuals, even though the room itself can accommodate up to 10 individuals.

Research by Wigdor et al. (2009) further indicates that the setup of such a collaboration environment must allow meeting participants to have equal opportunity for contributing to the discussion, preventing a single user from exclusively controlling the system and consequently also the conversation. Aiming to facilitate and encourage user interaction, the centerpiece of the hardware infrastructure is a multi-touch screen that makes it possible for all individuals to share, visualize and discuss digital content. The multi-touch feature intends to encourage participation as it is a direct invitation to take control compared to for example a traditional computer control based on a mouse and a keyboard. In such setups, the person currently in control can find it difficult to hand over control to the others, and the others find it awkward to 
ask or take control (Rogers et al. 2009). The touch screen is oriented horizontally as a tabletop to further encourage users to participate in collaborative activities (Rogers \& Lindley, 2004).

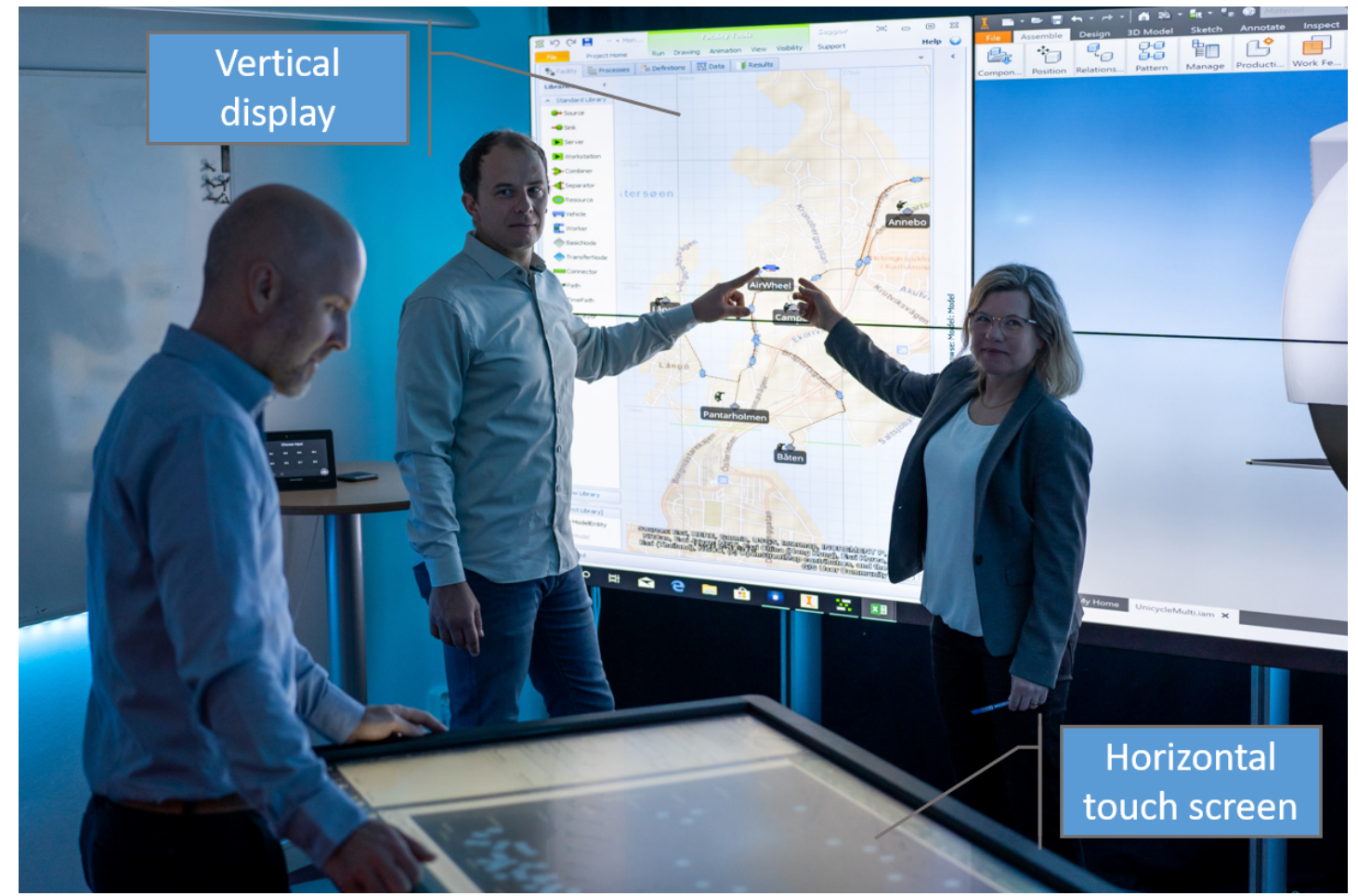

Figure 1. Overview of the Decision Arena.

In addition to the horizontal touch screen, the room also contains a large vertical display. This is intended as a canvas for displaying a variety of items such as concept descriptions, models and simulation results. Visualization is an important part of the intended workflow as it may augment problem-solving capabilities by enabling the processing of more data without overloading the user (Ware, 2005). Vertical displays are superior for audience-based viewing (Rogers \& Lindley, 2004) and allow the group to see information from the same perspective in contrast to a tabletop display. Furthermore, they are found to increase performance on spatial tasks such as 3D navigation (Tan et al., 2006). The video 'wall' consist of six 55-inch Full HD screens mounted in a three by two matrix. Using a video wall implies a natural way to display several sources simultaneously, providing mechanisms enabling content sharing of selected material between personal workspaces like laptops and the shared workspace. The main intention of this layout is to create a climate where participants can share material or applications without giving up ownership of the shared content. The room also contains traditional analog media such as two whiteboards that are mounted on opposite sides of the room. The intent here is to avoid situations where one individual takes control over the board making it challenging for others to take the floor. Even though the act of transforming ink-based information to a digital media is time and recourse consuming (e.g., due to the need of transcribing or taking pictures for the purpose of documenting/sharing/archiving), analog media have been found to be more usable and accessible and to have a positive impact on fueling the design discussions. Noticeably, although the environment is intended for collocated meetings, a video conferencing system is added allowing participants to join meetings remotely.

\subsection{The DA model-driven digital space}

The digital space of the DA is designed to support the team in harvesting, generating, structuring, storing and analyzing data to enable efficient information flows and increasing the 
users' ability to understand unstructured problems. The main aspect of the design of the digital space is how to leverage the ability of the team to handle design situations that are novel, nonroutine, not well understood and/or not agreed upon. This is because the design process is about generating a product and/or service that satisfy needs in the best way within available means. Yet, needs and available means are often ill-defined and conflicting during the early stages of design. In order to cope with ambiguity, uncertainty and the dynamic nature of early stage design knowledge, the DA exploits model-based experimentation (simulation) to enable extensive exploration, so to learn faster (by performing more and earlier iterations) about the characteristics of the best possible design (see: Thomke \& Fujimoto, 2000).

Systems Engineering (SE) research has stressed the importance of a specific model type to learn about what a 'best possible design' is: the value model (Collopy and Hollingsworth, 2011). This is expressed as a single objective function that aims at measuring the "goodness" of the design. The resulting value score, expressed in monetary units, becomes then the key decision-making criterion for the design team: the higher is the value, the more successful the solution will be. The core of the digital space of the DA relies then on a hybrid modeling environment with a multi-level model hierarchy - to support this Value Driven Design (VDD) approach (Bertoni and Bertoni, 2019). The environment encompasses, for example, discrete event simulations, finite element simulations, differential equations, algebraic equations and mathematical logic. At the same time, the DA proposes an automated approach for model-based exploration, in contrast with more traditional manual processes where computerized virtual models are exploited as 'islands' in the process. The component enabling this automation is the DA server. This controls interaction, data transfer and execution of all modules in the DA. The main modules are the functional model, the cost model and the value model respectively. Their functionality is based on submodules implemented in different software clients, whose interfaces are designed in the model framework. This allows models of different fidelity to be used in different stages of a project. Furthermore, this is important to easily swap between different projects. A thorough description of the digital space is given in Wall et al. (2018).

\section{Verification}

Verification activities were conducted to assess the ability of the DA to support decisionmaking activities in conceptual design. While qualitative feedback was obtained by demonstrating the physical environment to system experts and process owners, quantitative data were obtained through an experimental session with industrial practitioners. The physical space staged a design episode whose objective was to develop a concept for a battery-powered double-drum asphalt roller from an existing diesel-driven machine. The experiment involved five practitioners from a Swedish multinational engineering company that develops and manufactures pavers, mobile feeders, rollers and planers for asphalt compaction. The participants belonged to different organizational roles and functions, including product development, customer operations, marketing and finance. The experiment kicked-off with a 15-minute introduction on the DA functionalities and on the objective of the session. The experiment was conducted directly after this introduction and lasted for a total of 51 minutes. The goal was to determine the engineering characteristics (EC) of the electrical roller including battery technology, battery capacity and electric motor type - using as a basis an existing product platform. In the session, the team was able to explore the behavior of different combinations of these variables in the DA, modifying the design constraints and scenario data to identify the most valuable combination for the machine in two relevant applications (i.e., pothole and sidewalk compaction). Figure 2 shows the user interface featured in the experiment. The top-left corner of the interface displays information related to design constraints, such as volume and mass requirements. The bottom-left side allows the team to modify the EC of the 
system, so as to test the suitability of a design configuration in given scenarios. The latter are displayed (and manipulated) on the right-end side of the interface. The model results are displayed in the form of total energy consumption per day, battery cost and charging events.

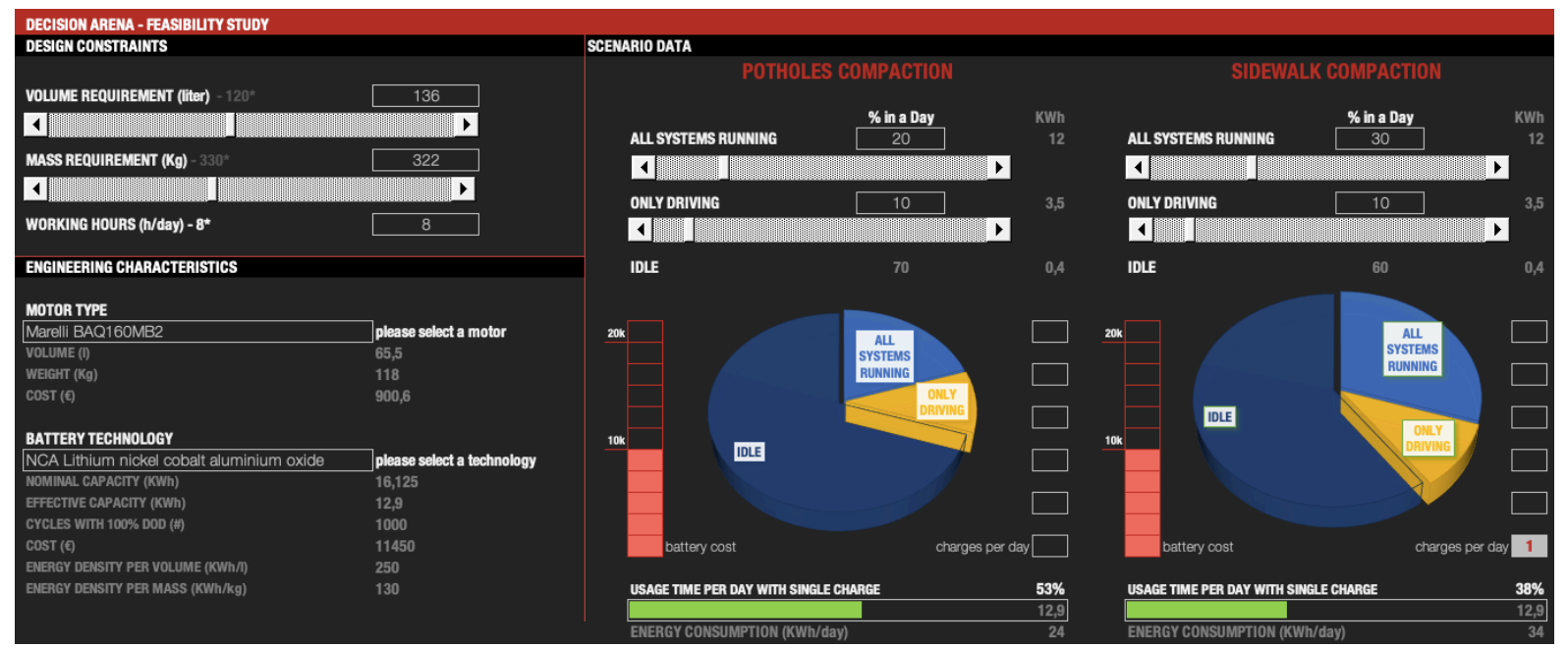

Figure 2: User interface featured in the design session (NW! data have been scaled to protect confidentiality)

The machine configurations were evaluated considering two alternative PSS business models: Product-oriented PSS (the machine is sold to the customer) and Use-Oriented (the hardware is part of renting agreement). At the end of the experiment, the researchers demonstrated how the selected EC could be used to calculate cost and value models. This session lasted for one hour and was facilitated by the researchers, helping the industrial practitioners in manipulating the modeling environment and guiding the discussion related to cost/value models results. The final step of the experimental activity featured a wrap-up session, where the main criticalities and lessons learned from the activity were discussed.

The conversation in the experiment was captured by two cameras with microphones positioned in two opposite corners of the room. These data were later transcribed and used to analyze the verbal interaction occurring in the experiment. Two main proxies were used to assess the ability of the environment to support cross-functional negotiation in the session. The authors initially measured the number of times each individual interacted with the other participants during the experiment. These 'interventions' were defined by the researchers as any meaningful contribution in spoken language that lasted at least 1 second. They typically include statements where the participants expressed ideas (e.g., "Can we increase the volume?"), opinions (e.g., "I do not agree!"), judgments (e.g., "Yes, I like it!") or any other argument. The analysis was deepened to map how the dialogue bounced between the different functions in the organization, by recording the total time each individual was active in the discussion.

\subsection{Experimental results}

The experimental session rendered a total of 517 interventions, which are almost equally distributed among each participating individual (Figure 3). The results show a good balance in the discussion, with each participant having the opportunity to voice his/her opinions, and to share knowledge with others. This distributed participation is interpreted as an indication that the environment invites for idea exchange, knowledge sharing and negotiation. 


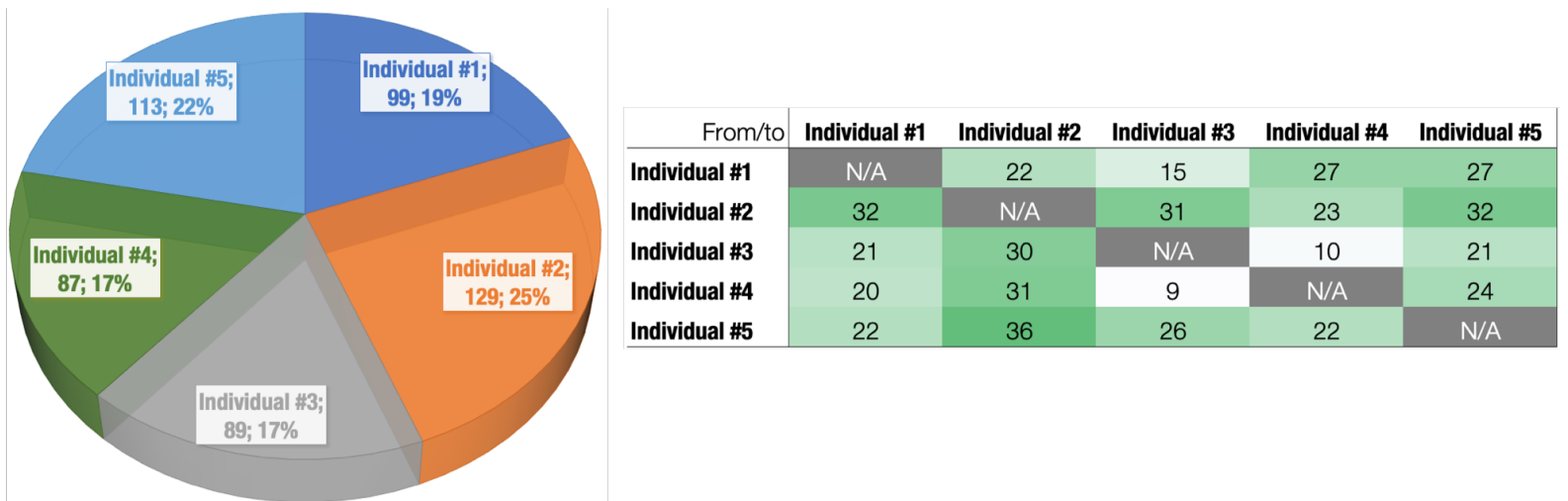

Figure 3: Number of interventions with percentages (left) and interaction map (right)

The interaction map on the right-end side of Figure 3, which details how the conversation bounced from a participant to another during the experiment, shows that the design session was not dominated by a single person, or by a single line of dialogue. Rather, all participants interacted with each other to a greater or lesser extent. This is interpreted by the researchers as an indication that no discipline or group (e.g., product developers) feels more at home than their counterparts in the physical and digital space. The latter does not seem to demarcate any real 'territory' but is rather seen as an enabler for work that is considered cross-disciplinary.
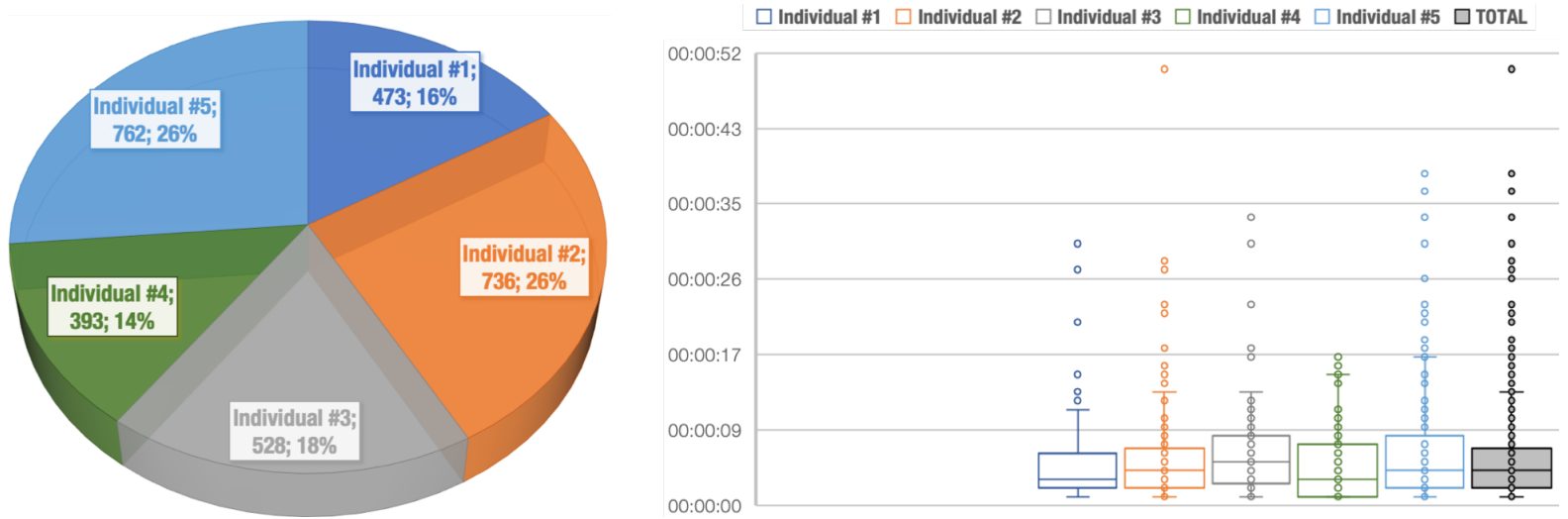

Figure 4: Total intervention time per individual with percentage (left) and exploded view (right)

Figure 4 shows the total intervention time divided for each individual, Also in this case, the results show a good balance in the conversation, with no discipline taking a leading role. These results are seen by the researchers as an indication that the environment supports a process where participants can quickly learn about dependencies and differences across organizational boundaries. This is testified by the high frequency of the interaction observed in the experiment. On average, each participant talked for about 6 seconds during the design session. Noticeably, the average intervention time is equally distributed among the group, ranging from a minimum of 5 seconds to a maximum of 7 for each individual. There is also evidence that the environment triggers deeper reflections on the meaning of the presented models and of the challenges related to them. On several occasions, a participant took the word for more than 30 seconds without being interrupted or questioned during the dialogue. This phenomenon points to the capability of the environment to support work that is not interdisciplinary, which is the DA makes it possible for individuals to adjust inputs into a description that features their own needs and triggers disciplinary-specific reflections.

The video from the experiment was coded to analyze the physical interaction between the participants and the physical space. The researchers observed to what extent the participants pointed to the information displayed on the video wall (as well as on the touch-screen) when creating arguments in favor (or against) a given design configuration. Also, the researchers 
observed how information displayed in the interface were manipulated by the team (e.g., by moving the sliders or inputting new values through the keyboard).
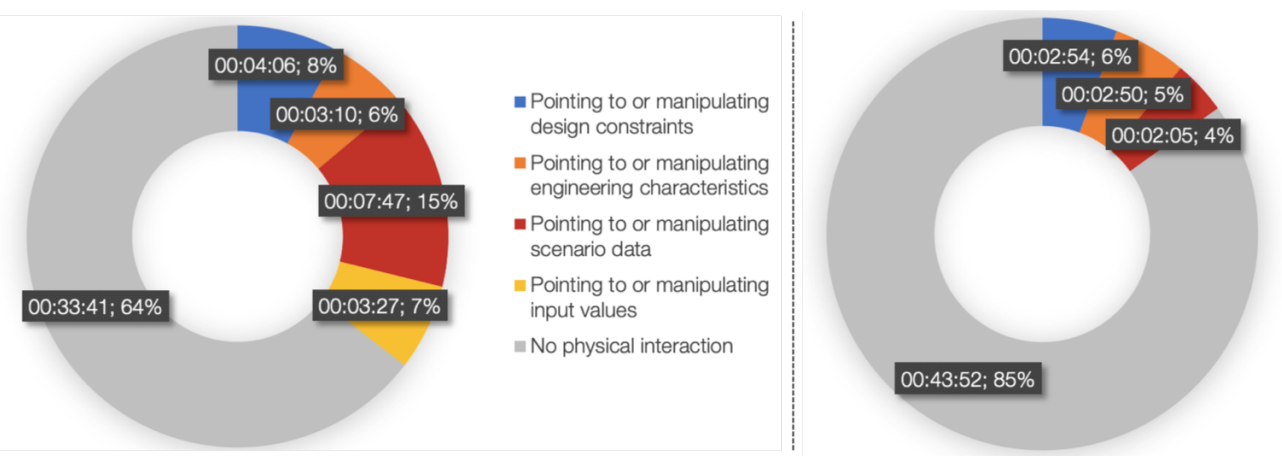

- Manipulating design constraints

- Manipulating engineering characteristics

- Manipulating scenario data

No manipulation

Figure 5: Overall time spent by the team pointing to or manipulating the information displayed by the user interface (left) or just manipulating it (right)

Figure 5 shows that the environment was used for more than $35 \%$ of the time to support the discussion and convey rationale and issues related to a design concept. At the same time, participants have actively utilized the environment for about $15 \%$ of the duration of the session (corresponding to approximatively 8 minutes) to manipulate the different input parameters for decision making. From a design perspective, it is interesting to observe that the Decision Arena was able to work as 'support' in all the areas described. In Figure 6, which is to convey a discussion both on the technical characteristics of a product, as well as on its value generation capabilities when applied in each scenario, the analysis was deepened to observe the extent to which the different individuals, corresponding to different roles and disciplines, were able to make use of the environment during the discussion. The left end side of Figure 6 shows the total number of times the participants point to the information displayed in the DA to justify a concept or an idea, or simply to ask for clarification from the other individuals.

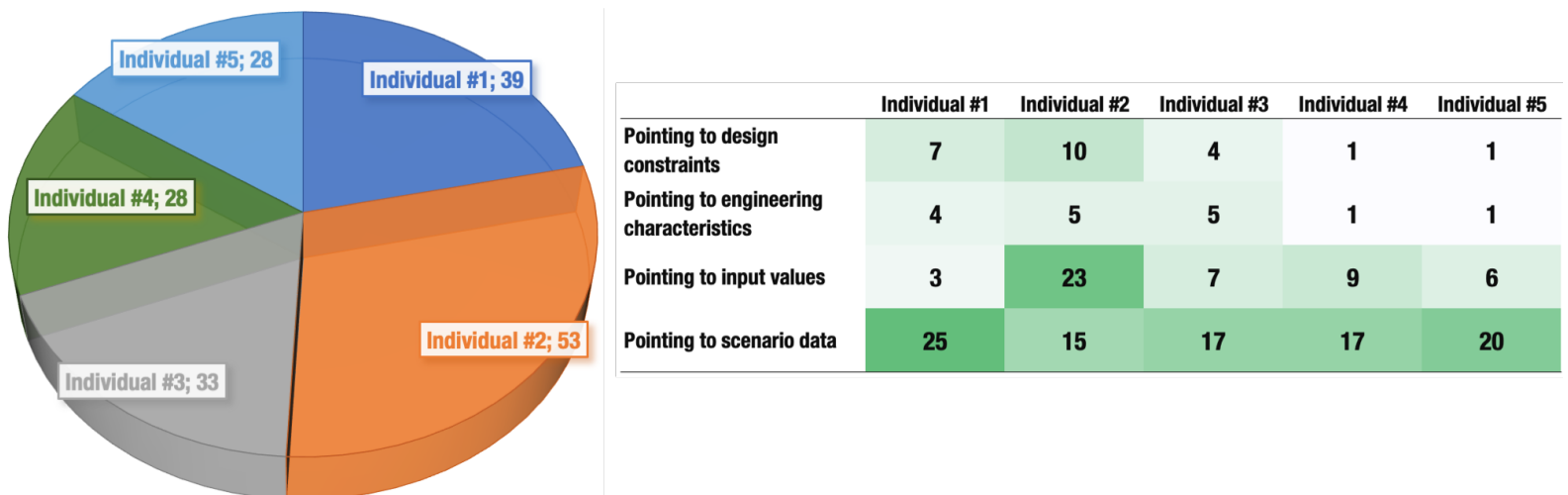

Figure 6: Number of times each individual pointed to the information featured by the user interface (left) with detailed mapping (right).

The participants interacted with the information displayed in the DA more than 170 times during the duration of the experiment. Indeed, all participants were seen to have interacted several times with the environment with little distinction, something that further indicates that no discipline or group feels more at home than their counterparts in the environment. A more detailed analysis, featured on the right end side of Figure 6, shows how the different individuals interacted with the environment with regards to the different areas featured in the interface. Also in this case, it is noticeable how each individual has interacted with each area at least once during the course of the experiment, further supporting the conclusion above. 


\section{Discussion and conclusions}

The paper presents findings related to the development of a model-driven environment for collaborative decision making in Product-Service System design, the Decision Arena (DA). The DA is characterized as a blended space whose main value resides in the interaction effect between the physical and the digital space. The rationale for the design of the DA, focusing on the physical space, is presented. Experimental sessions in ad-hoc design episodes were conducted to validate the design and intent of the DA. The experiment involved five practitioners in a cross-disciplinary setting, gathering individuals from different organizational roles and responsibilities including product development, customer operations, marketing, and finance. It was shown that all participants took an active part in addressing the design challenge, both looking at verbal communication and physical interaction with the physical as well as the digital space of the DA. This is, by the researchers, seen as confirmation that model-centric interactive group workspaces such as the DA, has the potential to augment collaborative design. Furthermore, the main ability of the DA is not only to facilitate decision-makers in exploring the design space using models but also to support negotiation in the cross-functional team, so as to facilitate the sharing of tacit, contextual knowledge about the product/service being designed. Several technical challenges emerged during the experiment, the main one being related to the speed of execution. The results of the simulation models need to be communicated to the design team almost instantaneously. However, in most cases, this is not attainable, and this was found to restrict discussions and negatively impacting knowledge sharing in the team. More than an issue of pure computing power, this is a problem of developing efficient schemes on how to produce data for the visualization, and how to manage them to enable a real-time experience when playing with design concepts. A way forward in this respect is that of creating surrogate models that could reduce computational time when working with computationallyheavy simulations, such as stress and deformation analysis and more.

\section{Acknowledgment}

The research leading to these results has received financial support from the Swedish Knowledge and Competence Development Foundation through the Model-Driven Development and Decision Support research profile at Blekinge Institute of Technology.

\section{References}

Benyon D. \& Mival, O. (2015). Blended Spaces for Collaboration, Computer Supported Cooperative Work (CSCW), 24:223-249

Bertoni, M. and Bertoni, A. (2019) Iterative value models generation in engineering design: lessons learned from a cross-company study. Design Science, Vol. 5, No. 18, 22p.

Bey, N. \& Mcaloone, T.C. (2006). From LCA to PSS - making leaps towards sustainability by applying product/ service-system thinking in product development. In 13th CIRP International Conference on Life Cycle Engineering, 571-576

Blessing, L. T., \& Chakrabarti, A. (2009). DRM: A Design Research Methodology. London: Springer.

Brown B.R. (2015). Advanced systems engineering and model philosophy, Proc. of the Conf. on Summer Computer Simulation, SummerSim, Chicago, IL, USA, July 26-29

Collopy, P.D. \& Hollingsworth, P.M. (2011). Value-driven design. J. Aircr. 48(3). pp. 749-759.

Craft, S. \& Garcia, Y. E., (2016). Interpersonal Media Used by Couples in Non-Proximal Romantic Relationships: Implications for Psychological Practice, In Emotions and Technology, Emotions, Technology, and Health, Academic Press, pp. 211-224

ElMaraghy et al., (2012). Complexity in engineering design and manufacturing, CIRP Annals - Manufacturing Technology 
Haller, M. et al. (2010). The NiCE Discussion Room: Integrating Paper and Digital Media to Support Collocated Group Meetings, Proc. of the SIGCHI Conference on Human Factors in Computing Systems, Atlanta, Georgia, USA - April 10-15, pp. 609-618

Isaksson, O., Bertoni, M., Hallstedt, S. and Lavesson, N. (2015), "Model Based Decision Support for Value and Sustainability in Product Development", Proc. of the 20th International Conference on Engineering Design - ICED 2015, Design Society, Milan, 2015, pp. 21-30.

Murakami, Y. (2016). Linking knowledge absorption and transmission toward innovation in R \& D organizations", in Proc. of the 17th European Conference on Knowledge Management, vol. 2016-January, Academic Conferences Limited, pp. 667-675

Nieminen, M.P., Tyllinen, M. and Runonen, M. (2013). Digital war room for design, Proc. of the 15th international conference on Human Interface and the Management of Information: information and interaction for learning, culture, collaboration and business, July 21-26, Las Vegas, NV.

Rogers, Y., \& Lindley, S. (2004). Collaborating around vertical and horizontal displays: Which way is best? Interacting With Computers, 16, 1133-1152.

Rogers, Y., Lim, Y-K, Hazlewood, W. R. \& Marshall, P. (2009). Equal Opportunities: Do Shareable Interfaces Promote More Group Participation Than Single User Displays?, Human-Computer Interaction, 24:1-2, 79-116

Rhodes, D.H. and Ross, A.M. (2016). A vision for human-model interaction in interactive model-centric systems engineering, In 26th Annual INCOSE International Symposium, Edinburgh. Scotland.

Sethi, R. (2000). New Product Quality and Product Development Teams, Journal of Marketing, 64(2), pp. 1-14.

Tan, D.S., Gergle, D., Scupelli, P., Pausch, R. (2006). Physically large displays improve performance on spatial tasks. ACM Trans. Comput.-Hum. Interact. 13(1), pp. 71-99

Thomke, S. and Fujimoto, T. (2000). The effect of "front-loading" problem-solving on product development performance, J. Prod. Innov. Management, 17(2), pp. 128-142.

Ulrich, K.T. \& Eppinger, S.D. (2012). Product design and development. (5. ed., International ed.) Boston, Mass.: McGraw-Hill/Irwin.

Wall, J., Bertoni, M., and Larsson T. (2018). A model-driven decision arena: Augmenting decision making in early design, Proceedings of NordDesign 2018, August 16th-18th Linköping.

Ware C. (2005). Visual Queries: The Foundation of Visual Thinking, In: Tergan SO., Keller T. (eds) Knowledge and Information Visualization. Lecture Notes in Computer Science, vol 3426. Springer, Berlin, Heidelberg.

White, D.D., Wutich, A.Y., Larson K.L. and Lant T. (2015). Water management decision makers' evaluations of uncertainty in a decision support system: the case of WaterSim in the Decision Theater, J Environ Plann Man, 58:4, pp. 616-630

Wigdor, D., Jiang, H., Forlines, C., Borkin, M. and Shen, C. (2009). WeSpace: the design development and deployment of a walk-up and share multi-surface visual collaboration system, In Proceedings of the SIGCHI Conference on Human Factors in Computing Systems (CHI '09), ACM, New York, NY, USA, pp. 1237-1246

Wohlin, C., (2014). Guidelines for snowballing in systematic literature studies and a replication in software engineering, in Proc. of the 18th International Conference on Evaluation and Assessment in Software Engineering, London, England, pp. 1-10.

Wang, L, Shen, W., Xie, H., Neelamkavil, J., Pardasani, A., (2002). Collaborative conceptual design - state of the art and future trends, Computer-Aided Design, 34(13), pp. 981-996

Yin, R. K. (2013). Case study research: Design and methods. Sage publications. 